\title{
DECISIÓN, TOTALIDAD Y RESTO. APUNTES SOBRE LA TEOLOGÍA POLÍTICA DE CARL SCHMITT ${ }^{1}$
}

\author{
Pablo Oyarzún R. ${ }^{2}$
}

\section{Resumen/Abstract}

Este ensayo propone una discusión de los fundamentos de la teología política de Carl Schmitt a partir de la articulación de los conceptos de decisión, excepción y soberanía que configuran la definición de esta última. El punto principal de la discusión, que intenta dar cuenta de la estructura general del argumento schmittiano, recae en la idea de que la norma y su normatividad no pueden fundamentar totalmente, es decir, "sin resto" (restloss) la decisión acerca de la efectiva eventualidad de un caso de excepción. La cuestión del resto -y el esbozo de una "lógica del resto" - en cuanto lo extra-legal permitiría reconocer la complejidad del círculo performativo y auto-fundante de la decisión soberana, produce dos "restos". Uno que sería la decisión soberana misma, al tiempo activamente dentro y fuera del orden legal, y otro que sería aquello que es lo exceptuado por la excepción, pasivamente incluido y excluido a la vez de dicho orden.

Palabras clave: soberanía, decisión, excepción, resto, teología política

DECISION, TOTALITY AND REST. NOTES ON THE POLITICAL THEOLOGY OF CARL SCHMITT

This essay proposes a discussion of the foundations of Carl Schmitt's political theology based on the articulation of the concepts of decision, exception and sovereignty that make up the definition of the latter. The

\footnotetext{
${ }^{1}$ Este ensayo data del año 2006. Es uno de los resultados del proyecto FONDECYT 1040530 "Figuras del poder. Contribuciones a una analítica filosófica del poder desde una perspectiva metafísico-estética", del cual el autor fue investigador responsable.

${ }^{2}$ Chileno, Universidad de Chile. E-mail: oyarzun.pablo@gmail.com
} 
main point of the discussion, which tries to give an account of the general structure of the Schmittian argument, it is based on the idea that the norm and its normativity cant not completely ground, that is, "without rest" (restloss), the decision about the possibility of an exceptional case. The question of the rest - and the outline of a "logic of the rest" -insofar as the extra-legal would allow to recognize the complexity of the performative and self-founding circle of the sovereign decision, produces two "remains". One that would be the sovereign decision same, at time actively inside and outside the legal order, and another that would be that which is excepted by the exception, passively included and excluded at the same time from of that order.

Keywords: sovereignty, decision, exception, rest, political theology

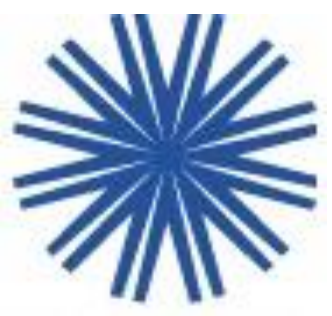

En 1922 publica Carl Schmitt un opúsculo bajo el título de Teología Política. ${ }^{3}$ Los "cuatro capítulos sobre la doctrina de la soberanía" que anuncia el subtítulo y que se despliegan en no más de setenta páginas proponen, de hecho, una reconstrucción de esa doctrina cuya clave teórica estriba en la recuperación de sus bases teológicas, y que entra en colisión frontal con la orientación dominante de la teoría jurídica de la época, presidida por la obra de Hans Kelsen.

\footnotetext{
${ }^{3}$ Para la edición que empleamos, v. la Bibliografía al final de este ensayo. La traducción de los pasajes citados es nuestra. Sin perjuicio de ello, referiremos a edición Héctor Orestes Aguilar (v. Bibliografía; la Teología política figura en las pp. 19-53 en traducción de Angelika Scherp). Señalamos en el texto las páginas respectivas de las dos ediciones empleadas, en primer lugar, la edición alemana y, separada por una barra oblicua, la edición mexicana). En caso de citar o referir otros textos de este libro, lo haremos bajo el nombre del editor.
} 
Ciertamente, bajo estos propósitos explícitos de envergadura teórica subyace una evaluación histórico-política de la modernidad, que ve en el liberalismo doctrinario una reducción esencial del sentido y el carácter mismo de lo político, coherente con el destino histórico de la modernidad, que Schmitt concibe bajo el concepto - relativamente estandarizado por esas fechas- de secularización. ${ }^{4}$

La intención de este trabajo es, si se quiere, hasta cierto punto formal. No estamos principalmente interesados en discutir los fundamentos y las implicaciones jurídicas de la enfática propuesta de Schmitt. Tampoco pretendemos discutir los apoyos que este busca en la tradición del pensamiento político y jurídico de la época moderna, que son invocados aquí como una suerte de memoria histórica tenaz de las raíces teológicas de la concepción del Estado. Lo que nos ocupará es, esencialmente, la lógica del argumento y, más precisamente, la lógica de los conceptos con que este se construye.

Pero, a pesar de lo limitada y escrupulosa que pueda parecer nuestra intención, no carece de importancia para la evaluación del significado de la concepción de Schmitt con vista a los debates contemporáneos en torno al devenir de la democracia.

\footnotetext{
${ }^{4}$ El término, que figura a propósito de una de las tesis principales del tratado, al comienzo de su tercer capítulo, es retomado por Schmitt en la Advertencia Preliminar a la segunda edición, fechada en 1933, y en la cual remite a su importante conferencia sobe "La época de las neutralizaciones y despolitizaciones", que había dictado en Barcelona cuatro años antes. Cf. Schmitt 2002, 79-95; v. también Schmitt 1984, 77-90.
} 
¿Una filosofía de la vida?

El párrafo conclusivo del primer capítulo de la Teología política reafirma desde perspectiva filosófica los elementos teóricos que integran la definición de la soberanía. En breves trazos queda perfilada una posición filosófica fundamental desde la cual se articularía la teoría del derecho que tiene en su centro dicha definición.

Precisamente una filosofía de la vida concreta (eine Philosophie des konkreten Lebens) no ha de retroceder ante la excepción y ante el caso extremo, sino que tiene que interesarse por él en la medida más alta. La excepción puede serle más importante que la regla, no en virtud de una ironía romántica que favorece lo paradójico, sino con toda la seriedad de una visión que mira más profundamente que las claras generalizaciones de lo que se repite estadísticamente (des durchschnittlich sich wiederholende). La excepción es más interesante que el caso normal. Lo normal no prueba nada, la excepción lo prueba todo (Das Normale beweist nichts, die Ausnahme beweist alles); no solo confirma la regla, la regla vive sin más solo de la excepción. En la excepción la fuerza de la vida real rompe la costra de un mecanismo encallecido en la repetición (In der Ausnahme durchbricht die Kraft des wirklichen Lebens die Kruste einer in Wiederholung erstarten Mechanik). (21 / 29)

La caracterización de esta "filosofía de la vida concreta" (real o efectiva), como base de la concepción del derecho contenida aquí, es en buena medida trivial, si la situamos en el contexto de las tendencias y discusiones contemporáneas a la redacción de la obra. La contraposición 
de la vida al mecanismo, a la repetición y al término medio es un tópico en esas discusiones, y si el planteamiento se restringiese a ella, se carecería de precisiones o especificaciones verdaderamente relevantes. Sin embargo, la vinculación con el tema de la excepción debe considerarse como el eje de la propuesta, la cual tiene como objetivo esencial restablecer el concepto de soberanía como concepto absoluto de la teoría constitucional y de la teoría del Estado. Es precisamente desde esta propuesta que se alza como tal eje el concepto de excepción. En cuanto que la filosofía de la vida por la que se aboga aquí tiene su interés supremo (y así también su medida esencial) en la excepción y en el caso extremo, es la vida misma la que se concibe como excepción y la que exige una conceptualización que se elabore desde el caso extremo o límite, es decir, una conceptualización que piense en conceptos límite.

Un segundo punto importante es la afirmación que sirve de fundamento a la tesis central del capítulo, y que se expresa de varios modos, de los cuales el más llamativo es el que señala que "lo normal no prueba nada, la excepción lo prueba todo" (ibíd.). En esta fórmula y en su continuación — "no solo confirma la regla, la regla vive en general solo de la excepción“ - viene a compendiarse la doctrina que sustenta la determinación de la soberanía. Por lo mismo, desde el punto de vista del argumento en que se entretejen las nociones fundamentales de este ensayo y los vínculos que se establecen entre ellas, más allá o más acá de los supuestos metafísicos a que están asociadas, es esencial ponderar la relación entre excepción y regla, entre el momento de extra-legalidad 
que en la concepción schmittiana caracteriza a la soberanía y el orden legal que ella funda.

\section{La definición}

La Teología política se abre con una sentencia celebérrima, de cuya concentrada energía - lindante, acaso, con la exasperación — hay pocos parangones en la literatura teórica del siglo veinte: "Soberano es quien decide sobre el estado de excepción. (Souverän ist, wer über den Ausnahmezustand entscheidet)." (13/23)

Tal es la "definición de la soberanía", según anuncia el título de este primer capítulo. El cuerpo del capítulo debe estar consagrado a dilucidar el carácter, alcance y contenido de la definición, considerando el peso específico de cada uno de los términos que la integran. Por lo pronto, se observará que la definición se desplaza del término "soberanía" al término "soberano", poniendo el acento en el sujeto de la soberanía, como si no fuese procedente hablar de esta en términos meramente abstractos o formales, sino siempre en cuanto detentada y, si se quiere decirlo así, personificada. Es lo que más adelante calificaremos con el adjetivo "existencial". 5 Pero examinemos la definición en su composición estructural.

\footnotetext{
${ }^{5}$ En la aplicación de este término permanecemos atentos al uso que de él hace Schmitt. En particular, su significación en El concepto de lo político es de primera importancia, en la medida en que, también a título de valor o valencia límite, permite fundamentar la diferencia de amigo y enemigo que, como se sabe, es la diferencia categorial que propone el ensayo como criterio de lo específicamente político, como criterio que a la vez totaliza esta esfera y la proyecta como esfera total. El enemigo "[e]s precisamente el otro, el extraño (der Fremde), y basta a su esencia que sea existencialmente (existentiell) algo otro y extraño en un sentido especialmente intensivo, de modo que
} 
En primer lugar, se trata del estatuto del concepto que se define. Soberanía es un "concepto límite" (Grenzbegriff). El sentido —o el valor - de lo que aquí se llama "límite" no queda inmediatamente claro. Se estipula que un "concepto límite" no es un concepto confuso, sino "un concepto de la esfera más extrema (äußersten Sphäre)" (ibíd.). La estipulación introduce una relación formal entre límite y extremidad, en virtud de la cual lo definido se sustrae a todo aquello que se incluya como un miembro más al interior de la "esfera”, implicando así que el estatuto ontológico de la soberanía es sui generis. Es lo que señala la continuación en cuanto explica que la definición de la soberanía no puede arrancar del "caso normal" (Normalfall), sino de un "caso límite" (Grenzfall) (ibíd.). Desde el punto de vista formal, el primero es lo que llamamos un miembro más de la esfera, que comparte con los otros las mismas notas o características de pertenencia a la esfera y de conformidad con su legalidad, en tanto que el segundo es (o sería) aquel que revela la condición de la esfera como tal. Es, desde la perspectiva de

en el caso extremo sean posibles con él conflictos que no puedan ser decididos ni a través de un sistema de normas preestablecidas, ni a través del dictamen de un tercero «no comprometido»y por eso «imparcial»." (Schmitt 2002, 27; cf. también Orestes 2004, 177, de donde tomamos la traducción con algunas modificaciones). El sesgo performativo (ciertamente, no normativo) de esta definición del enemigo, fundada en su extrañeza existencial, sesgo que parece enteramente coherente con el decisionismo de Schmitt, es acentuado por la continuación: "El caso conflictivo extremo solo lo pueden resolver entre sí los partícipes; en particular, solo por sí mismo puede decidir cada uno de ellos si el ser otro del extraño significa en el caso conflictivo concretamente dado la negación de la propia existencia y por eso ha de oponerse defensa o combatir para preservar el modo de vida propio, esencial (seinsmäßig)" (la traducción es nuestra). Para una discusión de esta definición y, en general, de la diferencia de amigo y enemigo, cf. Berndt 2003, 55 ss. 
la lógica con que se construye la definición, lo propio de algo que podríamos llamar el "principio de la extremidad".

Este "caso límite" es, para la teoría del derecho, en cuya estructura se busca validar la soberanía como concepto fundamental y fundacional, el “estado de excepción” (Ausnahmezustand). También formalmente, es la relación entre soberanía y estado de excepción la determinación esencial del concepto que se trata de definir. Esto supone una comprensión del estado de excepción que lo reconozca en su especificidad, es decir, en su diferencia respecto de otros conceptos afines, como son el "estado de emergencia" (Notzustand) y el "estado de sitio" (Belagerungszustand). Todo ocurre como si, desde el punto de vista lógico, el concepto de estado de excepción solo pudiera asirse en la medida en que se lo exceptúa (se lo extrae, excapio) de toda relación con otros estados afines que están contemplados en la legislación. En correspondencia con ello, se lo designa como "el caso no circunscrito en el orden jurídico" (13 s. / 23) y se lo señala como un "concepto general (o universal: allgemeiner) de la teoría del Estado". En esta medida, se podría decir que la lógica de este concepto - lógica que debe precisar en qué sentido se habla aquí de generalidad o universalidad - responde a lo que podríamos llamar un “principio de excepción”. Decir que la excepción es un concepto general equivale a decir que es absoluto (así se habla de inmediato de una "excepción absoluta"), y lo absoluto es en este caso (que es el caso límite) aquello que se constituye por excepción. ${ }^{6}$

\footnotetext{
${ }^{6} \mathrm{O}$, para decirlo en términos afines a la idea de lo total, que apuntábamos de paso en una nota anterior, y que será tema en lo sucesivo: la "universalidad" o "generalidad" del concepto de "estado de excepción" consiste en que cubre potencialmente todo el
} 
En tercer lugar, la decisión (Entscheidung, Dezision): sobre ella recae de lleno todo lo que podríamos llamar el peso material (o, como decíamos, existencial) de la definición. La decisión es el acto que determina la excepción y que identifica al soberano. Pero es precisamente la "decisión sobre la excepción" (Entscheidung über die Ausnahme), la decisión que decide que está dado "un genuino caso de excepción”, la que caracteriza al soberano: ella es decisión en sentido eminente (im eminenten Sinne). La lógica que rige esta explicación de la decisión es enteramente congruente con lo que denominamos el principio de la extremidad, que es, como veíamos, si no idéntico, al menos solidario del principio de excepción. También aquí el concepto de decisión es construido como un concepto límite. No toda decisión es eminente, no toda decisión recae sobre la excepción; solo la decisión sobre la excepción es decisión diríamos ahora- en sentido absoluto, es decisión total y es totalmente decisión. En este sentido se habla luego de la "significación autónoma de la decisión" (selbständige Bedeutung der Dezision): selbständig es aquello que se sustenta en sí mismo y está, así, esencialmente separado (exceptuado) del resto; es, si así puede decirse para satisfacer la idea de autonomía, aquello que se da la ley a sí mismo y se da a sí mismo como ley.

Decimos que el concepto de decisión soporta el peso material o existencial de la definición. La decisión es un acto: este es un primer

ámbito del orden de lo político, es decir, que constituye, desde su situación limítrofe, la clave para la recta comprensión del Estado como tal. 
punto que importa tomar en consideración, en cuanto es el acto de un sujeto que se define como tal y en su carácter esencial por dicho acto. Soberano es quien decide en sentido eminente, total o absoluto: al decidir, se sustenta por sí, se actualiza como tal y se separa de todo otro sujeto, se separa de los sujetos "normales", es decir, de aquellos que están bajo la norma y la generalidad de las normas. Pero la decisión eminente lo es en cuanto tiene un objeto esencial, que es el estado de excepción. Si el soberano se determina (actualizándose) por la decisión, él mismo se decide como soberano en cuanto decide (sobre) el estado de excepción. Y ya debe estar claro que esta decisión decide también la exceptuación del soberano respecto de la condición que su misma decisión establece. La aclaración del concepto de (estado de) excepción es, pues, literalmente decisiva.

Antes de entrar en esta aclaración, conviene echar un vistazo a la lógica de la definición. Como ya se puede advertir, esta es una definición sui generis. Ciertamente no es una definición nominal y tampoco es normativa. No es nominal, porque no se limita a elucidar un concepto dado. No es normativa, en cuanto que la norma es insuficiente para dar el concepto de soberanía, porque no puede circunscribir la excepción: esta lo es, precisamente, respecto de la norma, por general que esta sea, y en esa medida la norma no puede fundamentar irrestrictamente la decisión (nicht restlos begründen, "sin resto", donde, por lo pronto, el "resto" es justamente la decisión misma) sobre si se da un caso excepcional genuino (ein echter Ausnahmefall). Por otra parte, la definición no es esencial, si por tal se entiende la delimitación de un ente 
mediante su referencia a otros conceptos superiores, por la misma razón que la distingue de una definición normativa. Es esencial, en la medida en que determina la esencia de la soberanía, pero esta determinación consiste en el acto mismo, en un sentido que podría ser asociable a la idea de que la existencia precede a la esencia. Vista desde el plano meramente lógico, la definición tiene, entonces, un aspecto circular, pero esta circularidad tiene que ver con la condición performativa que ella misma asume. Es más: podría decirse que la definición es propiamente una decisión, como si realizara en el discurso o como discurso la misma soberanía que define.

Las consideraciones que siguen en el texto parecen apoyar esta idea. Schmitt indica que la definición abstracta de la soberanía como "poder de dominio supremo, no derivado (höchste, nicht abgeleitete Herrschermacht)" es práctica y teóricamente indiferente, en tanto que lo litigioso es su "aplicación concreta" (13/23). La pregunta por esta aplicación es la pregunta por el quién: quién es soberano, quién es el que decide en el caso de conflicto. Pero esta pregunta solo puede ser respondida de cara al caso excepcional. En la medida en que este caso no puede ser circunscrito, delimitado, determinado objetivamente aunque se lo pueda caracterizar como extrema emergencia, riesgo de la existencia del Estado, crisis radical del orden público, etc.-, en la medida en que ante todo tiene que ser decidido como tal, remite al quién - al sujeto de la soberanía-, el cual se define a su vez precisamente por esta decisión. Esta es, entonces, la circularidad de que hablábamos: su pivote es la decisión, que actualiza de una sola vez ambos extremos. El 
punto para Schmitt es que la excepción no puede ser "deducida" de nada, así como tampoco puede determinarse de antemano qué hacer a su respecto. Ambas cosas son objeto de la decisión. La excepción es un factum absolutum, y la decisión es el acto por el cual este es absolutamente determinado como tal en conjunto con lo que ha de hacerse para enfrentarlo. De ahí surge, pues, el concepto efectivo -y originario- del soberano:

Él decide tanto sobre si el caso extremo de emergencia está dado (ob der extreme Notfall vorliegt), como también sobre lo que debe acontecer para apartarlo. Está fuera (außerhalb) del orden de derecho vigente y sin embargo pertenece a él (gehört doch zu ihr), pues es responsable por la decisión sobre si la constitución in toto puede ser suspendida. (14 / 24)

Esta fundamentación del concepto del soberano - que, en buenas cuentas, consiste en retrotraer la soberanía al fundamento de la decisión, en sí infundado, a la manera de un origen incondicionado e irreduciblees el contenido esencial de la definición. Pero la fundamentación implica un momento determinante: en cierto modo, el soberano está a la vez dentro y fuera del derecho. En virtud de su decisión, se pone fuera, concentrando en sí todos los poderes del Estado para hacerse cargo de la excepción que él mismo decide como tal; pero en cuanto responde por la decisión que lo pone a él mismo fuera (al suspender de plano la constitución), sigue perteneciendo a dicho orden: sigue esencialmente referido al orden. Esta caracterización sugiere que junto al principio de extremidad se debe contar también con el principio de una peculiar 
exterioridad, que ciertamente no es la exterioridad de lo que está fuera sin más, sino la exterioridad de lo limítrofe: sería precisamente en este sentido que habría que entender la idea del límite contenida en la especificación del concepto de soberanía como un "concepto límite". Pero a su vez extremidad y exterioridad deben ser referidos al "orden": en el límite en que se actualizan ambas en virtud de la decisión soberana el orden no es simplemente abolido, sino suspendido. Así, la vigencia y la existencia misma del orden quedan remitidas a la decisión soberana y al soberano que decide: dicho de otro modo — si es lícito formularlo así-, la existencia del orden depende aquí de la existencia del soberano, pero no en el sentido de la mera constancia fáctica de este último, sino del acto existencial supremo de la decisión.

La continuación del texto se aboca a una de las tesis centrales del libro, una vez establecido el concepto originario de soberanía, que se recoge en el aserto siguiente: "Todas las tendencias del desarrollo moderno del estado de derecho están encaminadas a dejar de lado al soberano en este sentido." $(14 / 24)$

Estas tendencias, sin embargo, presuponen la posibilidad de excluir radicalmente el caso excepcional extremo, lo cual ya no es una cuestión jurídica, sino, en última instancia, algo que depende de las convicciones filosófico-históricas o metafísicas de quien abogue por semejante eventualidad (ibíd.). 


\section{El estado de excepción}

Se ha dicho que el estado de excepción no es cualquier estado de emergencia o de sitio, y que es precisamente en este alcance que pertenece de manera esencial a la definición de la soberanía. A esto van dirigidas las observaciones de Schmitt acerca de la teoría de Bodin, que buscan mostrar, en contra de las "tendencias" antedichas, que la estrecha unión que hay entre ambos elementos —el concepto de soberanía y el caso excepcional- había sido tempranamente reconocida. Así, Bodin habría sido determinante en cuanto a concebir la soberanía en relación con el caso de emergencia (la nécessité), como unidad indivisible y como facultad de decidir la suspensión de la ley vigente. Así también, evocando su anterior obra sobre La Dictadura $(1921)^{7}$, sostiene Schmitt que los teóricos del derecho natural del siglo XVII habrían compartido esta visión que vincula soberanía, excepción y decisión. El punto a este respecto es la determinación de lo que sea orden público y seguridad. La convicción que Schmitt atribuye a esos teóricos y que intenta rescatar para su nueva fundamentación del concepto de soberanía es la de que el orden se funda como tal en la decisión.

Pues todo orden descansa sobre una decisión, y también el concepto del orden jurídico (Rechtsordnung), que se aplica sin pensarlo como algo que va de suyo, contiene en sí la oposición de los dos elementos distintos de lo jurídico. También el orden jurídico, como todo orden, descansa sobre una decisión y no sobre una norma. (16/25 s.)

\footnotetext{
${ }^{7}$ Cf. Schmitt 1928.
} 
Esta nueva aproximación al concepto de decisión es importante. Sin duda, faltan todavía las precisiones que Schmitt aportará en el segundo capítulo (y que estudiaremos más adelante), que permitirán medir la envergadura de esta noción, pero ya está claro, a partir de este nexo esencial entre decisión y orden, es decir, de la decisión soberana como determinación y establecimiento de un orden, que esa envergadura es total. Lo es en un sentido que todavía tendremos que examinar más de cerca, pero es posible entender desde aquí que la decisión mantiene una relación analógica con el acto creador divino. En tal medida, se podría pensar que la decisión de-cide entre orden y no-orden. Sin embargo, esto no pasa de ser una apariencia. La razón de esta apariencia estriba en que la decisión, en los términos en que la concibe Schmitt, efectivamente “crea" el orden, acaece en el límite entre dos "realidades" y se mantiene en ese límite o, más bien, la facultad de decidir define el límite en el cual se erige el soberano como tal.

El carácter y el alcance de la decisión no depende de la antítesis entre orden y no-orden (entre orden y caos): es, pues, de primera importancia entender cuáles son las dos "realidades" que la decisión deslinda. Ello se aclara cuando en la secuela del texto se determina conceptualmente y de manera fundamental el estado de excepción. Este consiste en una "facultad ilimitada (unbegrenzte Befugnis), es decir, [en] la suspensión de todo el orden vigente" (18 / 27). El efecto de semejante suspensión es teóricamente determinante: 
Los dos elementos del concepto "orden de derecho" (Rechts-ordnung, orden jurídico) se contraponen aquí y prueban su independencia conceptual. Tal como en el caso normal el momento autónomo de la decisión puede ser reducido a un mínimo, en el caso excepcional la norma es aniquilada. A pesar de ello, también el caso excepcional sigue siendo accesible al conocimiento jurídico, porque ambos elementos, tanto la norma como la decisión, permanecen dentro del marco de lo jurídico. (19/27)

Esta separación entre orden y derecho es el eje de la determinación conceptual de la excepción y, por ende, del acto que la decide: dicho de otro modo, la "decisión sobre el estado de excepción" es la de-cisión que separa y contrapone orden y derecho, generando originariamente un orden pasible de derecho. La verdadera antítesis que aquí está en juego es la antítesis entre orden y derecho. Particular claridad aporta en este contexto la introducción del concepto de poder (Macht), que no juega un papel sistemático en la exposición de Schmitt, pero cuya significación implícita debe ser considerada en vista de la mejor inteligencia de esta exposición. Poder y derecho no son igualados aquí. No es el poder el que hace el derecho, sino (como se dirá más tarde) la autoridad como reconocida instancia que garantiza el orden jurídico; si efectivamente ambos coinciden en la decisión soberana, esta solo tiene su sentido político originario en el establecimiento del Estado y del derecho y del estado de derecho; tal es la responsabilidad que asume el 
soberano con respecto a su decisión ${ }^{8}$. Lo que hace, pues, la decisión en el sentido esencial de una decisión sobre el estado de excepción- es actualizar una de-cisión primordial y a la vez salvar el hiato constitutivo que le es inherente. Es el hiato entre la idea del derecho (la forma y la norma como forma) y la contingencia del hecho.

Este punto es de primera importancia, y marca la significación sistemática — si puede decirse así — que corresponde otorgarle al "caso excepcional". Al mismo tiempo que "la excepción es lo no subsumible" (lo que también quiere decir lo que no es susceptible de ser traída a un marco de generalidad preestablecido, lo singular que ante todo esclarece lo general ${ }^{9}$ ), revela "en absoluta pureza" la decisión como "elemento formal jurídico específico" (19/27). Pero esta misma singularidad, que indica a la excepción como ese factum absolutum de que hablamos antes, es lo que la define como algo radicalmente distinto a una diferencia o divergencia dentro del sistema del derecho, es decir, una que se mantuviese bajo lo que podríamos denominar la normalidad de la norma. Esta, la normalidad de la norma, no es una simple implicación tautológica de lo que ya está contenido en el concepto de la norma en lo que concierne a su vigor para ordenar relaciones y comportamientos,

\footnotetext{
${ }^{8}$ La distinción entre poder y derecho es relevante también para entender que con ella se acredita la autonomía del derecho como forma y mecanismo orgánicos del orden.

${ }^{9}$ Recuérdese la cita de Kierkegaard con que concluye el primer capítulo, la cual aboga precisamente por el carácter determinante, evidenciador de la excepción: "La excepción explica lo general y a sí misma. Y si lo general pretende estudiarse correctamente, solo hay que buscar una verdadera excepción, la cual revela todo con mucha más claridad que lo general. [...] Si no hay explicación para [las excepciones], tampoco la habrá para lo general." (21/29).
} 
sino que apunta a la configuración de relaciones y comportamientos concretos (en la expresión de Schmitt: Lebensverhältnisse, relaciones vitales), configuración que ante todo debe ser establecida en términos de un "medio homogéneo" (homogenes Medium) que la norma debe presuponer inherentemente en vista de su vigencia. Lo que llamamos aquí "normalidad de la norma" es precisamente lo que Schmitt designa como orden, originariamente distinto al derecho. Pero el orden no se opone al derecho como lo carente de derecho: el orden es su posibilidad constitutiva. Schmitt señala que la norma "no es aplicable a un caos", lo que equivale a decir que la norma no tiene fuerza por sí misma para crear el orden sobre el cual debe regir. Este es el punto fundamental a partir del cual se determina la significación excepcional de la excepción y, con ella, de la decisión soberana. La excepción no es inmanente a lo regido en general y en particular por la norma (generalidad y particularidad que componen en su correspondencia aquel "medio homogéneo"), en la misma medida en que Schmitt construye el concepto de excepción como aquella diferencia irreducible que resiste toda integración al sistema, pero no por simple oposición — que equivaldría a algo así como la contrariedad entre orden y caos-, sino porque es la condición misma del sistema: "El caso excepcional ha aparecido en su figura absoluta cuando ante todo tiene que ser creada la situación en la cual puedan regir principios jurídicos." (19/28)

El concepto de "situación" (Situation) se inscribe, así como aquello que está implicado esencial y estructuralmente en la noción de excepción, precisamente allí donde esta traza la distinción y la oposición entre orden 
y derecho. "Tiene que establecerse el orden para que el orden jurídico tenga sentido. Tiene que ser creada una situación normal (normale Situation), y es soberano aquel que decide definitivamente acerca de si efectivamente rige este estado normal. Todo derecho es "derecho de situación.” (Ibíd.)

Tanto la forma y alcance del argumento de Schmitt como este concepto específico de situación merecen ser considerados con cautela. Por una parte, lo que se puede observar desde el punto de vista de la lógica del argumento es - así lo hemos sugerido antes, y la cita precedente a la que ahora abordamos lo deja enfáticamente en claro - un desplazamiento desde la idea del estado de excepción como una determinada suspensión del estado de derecho, en virtud de la cual el poder estatal enfrenta una circunstancia extrema de la vida política y social que pone en peligro la forma establecida de esta misma, la existencia del Estado u otra condición general similar, hacia la idea del estado de excepción como origen del orden social y político y condición de posibilidad de la aplicación de la norma jurídica. Esta última es su "figura absoluta".

Ciertamente, se puede pensar que Schmitt entiende que este desplazamiento está implícito en la noción misma de tal estado en cuanto se apela a esta para construir el concepto estricto de soberanía. Pero desde la perspectiva teórica la significación fundacional que se le confiere aquí a dicho estado no puede ser considerada simplemente como una consecuencia analítica de su concepto en cuanto está inscrito este en la matriz a que lo asocia Schmitt. En cierto sentido, podría decirse que el 
estado de excepción cobra aquí una significación análoga a la del contrato social en la teoría política moderna. Pero, a diferencia del valor teórico propio de este último - a título de ficción o hipótesis necesaria, el estado de excepción se inscribe en una frontera paradójica entre el valor teórico y la indicación de una facticidad concreta puesta en régimen: se observará el uso de la expresión "normalidad fáctica" para designar aquello que "no es meramente una «presuposición extrínseca»", sino que "pertenece [...] a [la] validez inmanente" de la norma (ibíd.). Esta significación paradójica y fronteriza es también, probablemente, lo que determina el alcance del concepto de "situación", que podría hacerse equivaler al régimen de lo que antes denominamos la "normalidad de la norma", como un estado de las "relaciones de vida" pasible de regulación normativa que requiere ante todo ser producido para que esta regulación pueda entrar en vigencia.

La generalidad de la "situación" es acentuada en el texto en cuanto se la concibe como producto de la decisión soberana: El soberano crea y garantiza la situación como un todo en su totalidad (Der Souverän schafft und garantiert die Situation als Ganzes in ihrer Totalität). Él tiene el monopolio de esta última decisión. En ello reside la esencia de la soberanía estatal, que, por tanto, en recto sentido, no ha de definirse como monopolio de la coerción o del dominio (Zwangs- oder Herrschaftsmonopol), sino como monopolio de la decisión (Entscheidungsmonopol), a propósito de lo cual se emplea la palabra decisión en el sentido universal que ulteriormente se desarrollará. El caso 
excepcional pone de manifiesto la esencia de la autoridad estatal del modo más claro. Aquí la decisión se separa de la norma jurídica, y (para formularlo paradójicamente) la autoridad demuestra que, para crear derecho, no necesita tener derecho (um Recht zu schaffen, nicht Recht zu haben braucht). (Ibíd.)

La contundente fórmula "la situación como un todo en su totalidad" devela a la vez el carácter originario de la soberanía y la decisión y de la decisión en la soberanía. Con ello también se alcanza la plenitud de la definición de la soberanía. Como se puede observar, el estado de excepción funciona en esta definición como pieza esencial, sobre la que recaen los valores de extremidad y exterioridad de que hablamos antes: es el "principio de excepción" lo que determina los valores de estos conceptos constituyéndolos en principios operantes en la definición. Pero vale la pena detenerse en aquello que, por complemento a lo dicho, se podría caracterizar como un "principio de totalidad", y que tal vez sería susceptible de ser identificado como el resultado de la lógica con que se construye esa definición. Si se concede esta lectura, siempre desde el plano formal, cabe observar que entre excepción y totalidad se tiende un vínculo fundamental. Para decirlo más específicamente, todo ocurre como si la excepción fuese la condición bajo cual la totalidad se hace no solo pensable, sino real en los hechos. La totalidad en cuanto tal, podría quizá precisarse. Porque la totalidad de la que se habla aquí es, por una parte, la totalidad efectiva de los hechos contingentes de la existencia colectiva o social — totalidad del ser social, si se quiere, que no requiere de la excepción para ser concebida ni de la decisión para existir, como 
quiera que se da en cada caso en el pormenor de su acaecer contingente, y, por otra, esa misma totalidad en cuanto efectivamente sometida a la norma jurídica, es decir, al deber ser en función al cual ha de organizarse esa multiplicidad empírica. Esta última es la totalidad en cuanto ordenada, y es la totalidad en cuanto tal en la medida en que solo ordenada tiene el carácter y el sentido de la totalidad. La totalidad oscila lógicamente, entonces, en la alternativa entre la mera contingencia (el des-orden) y el orden, y es exclusivamente el acto soberano de la decisión el que tiene la fuerza suficiente para salvar el hiato entre ambos (que la norma no puede cubrir por sí) instaurando en general el orden.

\section{La decisión}

La explicación de la soberanía ha dejado pendiente una aclaración del sentido que tiene en ella el término "decisión", que concierne a su envergadura universal. El segundo capítulo de la obra ("El problema de la soberanía como problema de la forma jurídica y de la decisión") se encarga de esto. Que lo haga a través de una discusión con el neokantismo de Kelsen y Krabbe (y de manera secundaria con Wolzendorff) señala ya hacia el carácter del concepto que propone Schmitt. Kelsen aparece aquí como el autor fundamental de una doctrina que afirma que "el fundamento de la validez de una norma solo puede ser a su vez una norma" (27 / 32), en última instancia la "norma fundamental" (Grundnorm) de Kelsen, erradicando así todo componente concreto, material o existencial, de dicho fundamento, toda causalidad ontológica, si puede decirse así, y garantizando lo que podríamos denominar el círculo formal de la fundamentación de la norma. Se 
desprende de ello que "el Estado es [...] para la consideración jurídica idéntico con su constitución, es decir, con la norma fundamental unitaria" (ibíd.). Schmitt entiende que esto equivale a solucionar el problema del concepto de soberanía mediante su negación, lo cual no refleja otra cosa que "la vieja negación liberal del Estado en contraposición al derecho y el desconocimiento del problema independiente de la realización del derecho (Rechtsverwirklichung)" (29 / 33), que tiene en Krabbe a uno de sus representantes recientes más importantes. A través del debate con el neokantismo se trae al primer plano la confrontación principal con el liberalismo.

El "problema independiente", es decir, irreducible de que habla Schmitt es la premisa esencial de su explicación de la decisión, que queda enunciada al comienzo del capítulo: "La combinación de poder supremo fáctico y jurídico es el problema fundamental del concepto de soberanía" (26 / 31). En dicha combinación recae la citada cuestión de la "realización del derecho" (Verwirklichung des Rechts), y esta realización supone un momento que no puede ser formalizado en términos de la razón discursiva. El síntoma de ello es la atención que ha merecido históricamente el tema de la personalidad en la constitución de la autoridad jurídica, y que el formalismo liberal ha intentado extirpar afirmando la "validez impersonal de una norma impersonal" (35/38).

El punto - es decir, el concepto general de decisión - es desarrollado por Schmitt mediante el análisis de la decisión jurídica y de su independencia respecto de toda consideración de contenido normativo, 
ya sea el contenido de la idea del derecho, ya el de una norma positiva general del derecho con ocasión de su aplicación:

Cada decisión jurídica conlleva un momento de indiferencia respecto del contenido (inhaltlicher Indifferenz), porque la conclusión jurídica no puede ser derivada hasta el último resto a partir de sus premisas, y la circunstancia de que es necesaria una decisión permanece como un momento independiente determinante. A este propósito, no se trata del surgimiento causal y psicológico de una decisión semejante, si bien también para esto es importante la decisión abstracta, sino de la determinación del valor jurídico. (36 / 39)

La necesidad de la decisión se inscribe en una suerte de punto ciego: la relación de la norma general con el caso particular, en la cual esta particularidad, si cabe decirlo así, ejerce como tal resistencia a la aplicación inmediata de la norma conforme a su contenido. Se podría argüir quizá que esta imposible inmediatez de la subsunción del caso a la norma es precisamente lo que viene a ser sustituido por la inmediatez de la decisión. Lo propio de lo normativo, sostiene Schmitt, es que "un hecho concreto tiene que ser juzgado concretamente, si bien solo está dado como criterio del enjuiciamiento un principio jurídico en su universalidad genérica" (37 / 39). En consecuencia, siempre hay aquí una "transformación", que no depende de la idea del derecho sino de una "auctoritatis interpositio". Esta interposición es equivalente a una interrupción radical del proceso discursivo $-\mathrm{y}$ del universo 
discursivo - en que subsiste y se despliega la norma y lo normativo, una interrupción que adquiere fisonomía personal.

Los comentarios de Schmitt dejan en claro que la intención que subyace al discernimiento de este "momento" no tiene que ver con la reivindicación de algún tipo de irracionalidad de la decisión jurídica frente a la racionalidad del sistema normativo. Lo que está en juego es el reconocimiento del calibre metafísico y, más precisamente, teológico del acto soberano:

El que fuese la instancia competente la que tomó la decisión hace a esta independiente de manera relativa, y eventualmente también de manera absoluta, de la corrección de su contenido y corta toda discusión ulterior acerca de si puedan subsistir dudas. La decisión se vuelve en el instante independiente de la fundamentación argumentativa y recibe un valor autónomo (selbständigen Wert). [...] Visto desde el contenido de la norma que está a la base, ese momento constitutivo, específico de decisión es algo nuevo y ajeno. La decisión, considerada normativamente, ha nacido de una nada. La fuerza jurídica de la decisión es algo distinto al resultado de la fundamentación. No se la atribuye con la ayuda de una norma, sino al revés; solo desde un punto de atribución se determina qué es una norma y qué es corrección normativa. (37 / 40)

El calibre de que hablamos queda claramente establecido en la sentencia que hace de eje de lo que aquí se dice: Die Entscheidung ist, normativ betrachtet, aus einem Nichts geboren. La sentencia especifica, si así 
puede decirse, el sentido en que la decisión es absolutamente diferente a un proceso o un recurso de fundamentación. Estos últimos, como ya lo adelantamos a propósito de la crítica de Schmitt a Kelsen, no hacen otra cosa que remontar de norma en norma hasta la "norma fundamental": la fundamentación queda encerrada en la inmanencia del universo normativo. Pero este universo es inane -es meramente formal y abstracto - en tanto no haya un orden sobre el cual se ejerzan las normas. Solo la decisión tiene la fuerza para imponer ese orden, que, por lo pronto, es cualquier orden o, si se quiere, en términos genéricos, es $e l$ principio del orden. Esta imposición, en cuanto es ajena a la economía de la fundamentación, es radicalmente infundada: "ha nacido de una nada". En este rasgo podemos reconocer por primera vez en el curso de nuestro análisis la vinculación esencial entre decisión, violencia y mundo. Lo que llamamos hace un momento "el principio del orden" que Schmitt afirma por sobre todo orden concreto- puede igualarse, desde el punto de vista teórico, con lo que denominamos "mundo", con el mero "haber mundo", que en lo sucesivo podrá organizarse en términos y regiones de sentido en virtud de las relaciones vitales y de las normas, cualesquiera que ellas sean, que han de regirlas. ${ }^{10}$ Por su parte, el acto mismo de la imposición es en sí violento, si por violencia vamos

\footnotetext{
${ }^{10}$ Regiones categoriales relativamente autónomas (relativ selbständige Sachgebieten) tales como las que describe Schmitt en El concepto de lo político: lo moral (bueno y malo), lo estético (bello y feo), lo económico (útil y dañino, rentable y no rentable). A diferencia de ellas, lo político, organizado específicamente según la diferencia entre amigo y enemigo, no tiene, sin embargo, ninguna región temática propiamente dicha, a cambio de lo cual puede constitutivamente extraer elementos de las otras tres en refuerzo del conflicto, de modo que virtualmente abarca la totalidad de la existencia social y virtualmente grava las valoraciones por las que se rige. Cf. Schmitt 2002, $26-$ 28; v. también Orestes 2004, 176-178.
} 
a entender aquello que excede y suprime por su propia excedencia todo proceso de fundamentación, es decir, toda justificación de lo existente en virtud de la representación de su razón de ser. La decisión sustituye anticipatoriamente toda razón de ser, y solo puede tener su propia razón en sí misma. Dicho de otro modo, y ahora en términos jurídicos, la decisión da cuenta circularmente de su legitimidad mediante la referencia al orden que ella misma impone.

Aquí también podemos observar lo que caracteriza a la lógica peculiar de la definición de la soberanía, que habíamos intentado poner de manifiesto más atrás. Pero lo importante es que esta circularidad "autofundante" no es sin resto. La lógica de la circularidad, que, como dijimos antes, es una circularidad performativa (cifrada en el acto de la decisión), depende, si podemos decirlo así, de la "lógica del resto". Cuando Schmitt, apenas iniciada la exposición, afirma que "una norma general [...] no puede jamás aprehender una excepción absoluta y, por eso, tampoco [puede] fundamentar sin resto (restlos) la decisión de que un genuino caso excepcional está dado" $(13 / 23)^{11}$, indica hacia este nexo esencial. Al comentar este pasaje, dijimos en su oportunidad que el resto en cuestión es la decisión misma. Ahora podemos mirar quizá con más precisión en el nexo, y observar que el "resto" no es solamente la decisión que, en su condición absoluta de infundada, es precisamente lo que se sustrae esencialmente a la economía de la fundamentación

11 La traducción castellana a que hemos referido vierte restlos por "totalmente", perdiéndose este, si podemos decirlo así, punctum del texto. 
normativa, sino que es también la excepción misma y así también la condición de la soberanía como tal.

Esta función del resto exige, entonces, ser comprendida con extremo cuidado. Digámoslo en términos generales: el "resto" es lo extra-legal por definición. ${ }^{12}$ En este sentido, y en primer término, determina la esencia de la decisión, de la excepción y de la soberanía, en cuanto solo por la decisión soberana que determina y establece la excepción como condición del orden puede advenir este, instaurando lo que llamamos la "normalidad de la norma". Sin embargo, este es solo un primer sentido de la extra-legalidad del "resto", por el cual el soberano reserva para sí positivamente su propia condición de exceptuado respecto de la legalidad que funda con su decisión ("la autoridad prueba que, para crear derecho, no requiere tener derecho", 19 / 28), sin por ello dejar de pertenecer a dicha legalidad, en virtud de esa misma decisión (“está fuera del orden jurídico normalmente vigente y sin embargo pertenece a él, porque es responsable por la decisión sobre si la constitución puede ser suspendida in toto", $14 / 24^{13}$ ). En un segundo sentido, el "resto" es lo

\footnotetext{
${ }^{12}$ La "extra-legalidad" es también el principio que explica la tesis schmittiana según la cual la relación política primigenia es la relación amigo-enemigo, tal como se la presenta en El concepto de lo político. Esta relación es anterior a toda ley y asimismo al Estado, según estipula famosamente la obertura del opúsculo con tajante aserto: "el concepto del Estado — dice Schmitt— presupone el concepto de lo político" (Schmitt 2002, 20; Orestes 2004 171), de modo que aquel no es otra cosa que la forma bajo la cual se organiza y canaliza la lucha entre amigo y enemigo. Tal como en la Teología política la decisión se opone radicalmente a la discusión (como proceder fundamental del liberalismo parlamentario), la relación amigo-enemigo excluye toda discusión y toda instancia de transacción en el acuerdo, y solo deja espacio, con su carga existencial, a la decisión.

${ }^{13}$ La pertenencia ciertamente no elimina la exterioridad del soberano: ella está, por decirlo así, conservada en dicha pertenencia, en la medida en que el soberano se define
} 
que la decisión soberana separa como aquello que, estando fuera de la legalidad vigente, pertenece no obstante a ella como lo que debe ser necesariamente exceptuado a fin de asegurar la vigencia de esa legalidad. La idea del ghetto, de la reducción, está cerca de este segundo sentido y probablemente entrega su clave.

El círculo auto-fundante y auto-referente de la decisión soberana se traza, pues, solo a condición de exceptuar dos "restos", uno de los cuales es la decisión soberana misma, que prevalece en el límite del círculo asegurando el orden de lo contenido en él, en tanto que el otro es lo exceptuado por la excepción como límite enclaustrado en el interior del orden legal sin derecho. Esta exención de derecho es, por supuesto, simétricamente inversa a aquella de la que goza el soberano. En el caso de este, el "no tener derecho" equivale a su facultad originaria de "crear derecho"; en el segundo caso, el "no tener derecho" equivale a la absoluta sumisión al derecho establecido sin facultad alguna.

Hay, pues, en una y la misma excepción, dos modos de operar de la excepción. Ambos incluyen y excluyen a la vez. Pero en uno de ellos el soberano es excluido de todo lo que queda incluido en el orden que crea de modo tal que él mismo queda incluido en este como aquello que activamente se excluye a sí mismo del derecho que rige ese orden. ${ }^{14}$ En

no solo por el acto de decidir, sino también en todo momento por la capacidad de decidir.

${ }^{14}$ Este, a nuestro parecer, es el sentido que tiene la insistencia de Schmitt en que la decisión y la soberanía, a despecho de su peculiaridad radical, siguen perteneciendo a la dimensión del derecho. 
el otro, el resto es incluido absolutamente en el orden como aquello que es simultáneamente excluido de tal derecho en cuanto miembro radicalmente pasivo (negativo, si se quiere) de su régimen.

Se dibuja así la extraña figura de un círculo deformado por la tensión entre los dos "restos" polares desde los cuales se traza, en el extremo superior, como un ápice que tiende a excederlo y trascenderlo, en el soberano, y, en el inferior, como una depresión interna que tiende a rizarse y cerrarse sobre sí, como una suerte de inmanencia anómala en la inmanencia del orden normalizado. Este círculo, que circunscribe el ámbito del derecho, sería lo que desde el punto de vista schmittiano cabe caracterizar como la totalidad.

En cierto modo, podríamos llamar a la lógica que gobierna todo este trazado y, por ende, la definición schmittiana de la soberanía como una lógica del tercero excluido. Como se sabe, el principio del tercero excluido (tertium non datur) se agrega, en la lógica clásica, al principio de identidad y al principio de no-contradicción, considerados como matrices fundamentales de la forma de todo pensamiento. Mientras el primero establece que algo es idéntico numérica, específica o genéricamente, el segundo determina que a algo no puede convenirle y no convenirle al mismo tiempo a una misma cosa; el tercero dice que una de las partes de la contradicción tiene que ser necesariamente verdadera y la otra falsa. Aristóteles perfiló estos principios; mencionó el primero, le dedicó una larga consideración al segundo y, aunque estableció el tercero, restringió su validez al pasado y al presente (donde el ser o no- 
ser de los entes ya está decidido), y no lo estimó aplicable a los acontecimientos futuros contingentes, es decir, a la mayor urdimbre del tejido que llamamos "vida". El tercer principio excluye todo tercero dondequiera que se presente una contradicción, cerrando así sobre sí mismo el gran formato binario del logos occidental, circunscribiendo su región, el espacio jurisdiccional que le es propio: la universalidad o, para decirlo en los términos en que hemos hablado del planteamiento schmittiano, la normalidad de la norma.

Si la universalidad del lenguaje y del pensamiento es posible a partir del principio lógico del tercero excluido, la totalidad de lo social bajo la norma solo es posible a partir del principio jurídico-político del tercero exceptuado.

Pero lo peculiar de esta lógica del tercer principio — que habría que reconstruir pacientemente como lógica de la excepción - no estriba simplemente en que la excepción admita dos modos de operar, como decíamos hace un momento. Los dos modos en que la excepción se bifurca retornan sobre la excepción misma en la forma del "a la vez" de la inclusión y la exclusión. Este retorno genera — podríamos suponer, por cierto más allá de lo que concibe Schmitt, y como punto o punctum crítico de su teoría - unas figuras en que ambos momentos, la inclusión y la exclusión, se presentan a la vez sin poder ser interna y estructuralmente discernidos: piénsese en Edipo, en Cristo, en lo que la teoría política moderna llama el "pueblo", en un tipo absolutamente único - único cada vez- de soberanía que, de uno u otro modo, es una 
soberanía sacrificial, es decir, a la vez, un sacrificio soberano que sacrifica al soberano potencial (es decir, la potencia de este) para establecer la soberanía en el modo en que el derecho lo conoce y que Schmitt, aquí, recupera con su definición decisionista.

Sea lo que fuere de estas extrapolaciones, conviene rematar nuestra consideración de la decisión con la formalización que hace Schmitt de su postulación. El sentido teórico de la decisión aislada en su pureza, rescatada de su explicación meramente psicológica o sociológica, reconocida en su significación jurídica originaria, es consolidado por Schmitt mediante la hipótesis de "dos tipos de cientificidad jurídica", que se definen precisamente por ese mismo reconocimiento. Ello permite a Schmitt identificar, junto al tipo normativista que critica, un tipo decisionista (dezisionistisch), cuyo representante clásico es, según él, Hobbes, que ha formulado la antítesis fundamental: auctoritas, non veritas facet legem (cf. 39 / 41). Si la veritas define la idea o la forma ideal del derecho, que, como se ha visto, no tiene la fuerza para ponerse a sí misma como principio del orden, la auctoritas es el poder soberano que en la concreción y en virtud de su acto decisorio primordial, que se pone a sí mismo como tal principio y origen, genera el orden de la existencia social, susceptible ahora de regimentación jurídica. La idea hobbesiana de hacer prevalecer el principio del orden sobre cualquier tipo de orden concreto encuentra en el apelativo de decisionista su carácter teorético. Pero tal vez esta ecuación entre orden y decisión, articulada por el personalismo que Hobbes postula y que Schmitt celebra, no sea la única posibilidad lógica del decisionismo en cuestión. 


\section{Teología política}

El título del tratado es también el encabezado de su tercer capítulo. Se inicia este con otro de los asertos programáticos de Schmitt: "Todos los conceptos significativos (prägnante) de la doctrina moderna del Estado son conceptos teológicos secularizados." (44 / 43)

Enunciados de esta naturaleza, cuya contundencia ya hemos señalado a propósito de otros precedentes al que ahora leemos, escanden el texto dando cuenta también de su lógica profunda, es decir, no solo del orden secuencial de la exposición —el orden de las razones que sustenta el argumento-, sino del modo de pensamiento peculiar de Schmitt, que ya se anuncia con su temprana llamada de atención sobre los conceptoslímite. Este modo —el del pensamiento del límite y en el límitedestaca precisamente asertos como el que ahora enfrentamos con un rasgo axiomático que no puede ser reducido exclusivamente a los procesos inferenciales del discurso argumentativo, sino que toca su frontera - es decir, el conjunto de condiciones de posibilidad que Schmitt reconoce como únicas que son necesarias y suficientes para la articulación de una teoría política apta para pensar el estatuto de lo político, del Estado y del derecho en un momento histórico para el cual las formas de pensar que mantienen vigencia en la modernidad (ante todo, aquellas que han sido acuñadas en la matriz del liberalismo) ya no son adecuadas- - Por esa misma razón, tales asertos son como saltos en el curso del razonamiento — verdaderas instancias de decisión, ya se ha sugerido-, en virtud de los cuales este puede hacer pie, se diría, más en el temple que los dicta (la "pasión enérgica" invocada por Schmitt 
mediante el expediente de la cita de Kierkegaard) que en los puros títulos de racionalidad que los autorizarían.

En el caso del que ahora nos ocupa, se trata de un enunciado que identifica la tendencia general de este pensamiento desde el punto de vista teorético. Esa tendencia apunta a la vez a una crítica de la teoría moderna del Estado y a una restitución de sus premisas básicas, las cuales, sin embargo, permanecerían ocultas, obliteradas o latentes, en la armazón de esa misma teoría, en cuanto ella se sostiene en ciertos conceptos fundamentales y en sus implicaciones sistemáticas. Esa restitución es la de la teología política como fundamento de la teoría moderna del Estado. Podemos suponer que con ella no se pretende tanto un retorno a las formas medievales de concepción del Estado y la soberanía, sino llevar a cabo un despeje crítico que haga posible la reconstrucción de dicha teoría a fin de satisfacer las exigencias que plantean las transformaciones contemporáneas de lo político. Si se puede caracterizar la postura de Schmitt como la de un conservadurismo estricto, este va asociado a una perspectiva que es, al menos desde el punto de vista teórico, radicalmente revolucionaria. El punto de convergencia de ambos aspectos del planteamiento de Schmitt está dado por la idea de orden. La refacción profunda de la teoría política está al servicio de la comprensión de los principios en virtud de los cuales puede ser efectivamente establecido y preservado el orden en una sociedad cuyas estructuras y procesos se han transformado decisivamente en el último siglo. 
Y este punto es de primera importancia, porque, junto con sentar la actualidad enteramente incidente que tuvo la reflexión de Schmitt en el momento de su formulación, explica entre otras cosas la enorme atracción que han suscitado sus planteamientos entre teóricos de diversa procedencia y posición.

El enunciado en cuestión contiene un término que supone un compromiso teórico e interpretativo. La idea de la secularización lleva consigo una determinada comprensión de la modernidad, que la entiende como un proceso en virtud del cual se debilita hasta el punto de su supresión toda instancia de trascendencia entendida como fundamento del mundo y de su orden y se tiende a concebir a este último como un orden regulado de manera inmanente, a la manera de la imagen que de la naturaleza se hacen las dominantes ciencias naturales. Si el trasfondo metafísico-teológico del concepto de soberanía es bastante obvio, en la medida en que el soberano absoluto que establece el orden legal es concebible por analogía con el Dios creador omnipotente (recuérdense, a este propósito, las caracterizaciones que Schmitt ofrece del acto decisorio), la secularización no puede sino importar la tendencia a la erradicación de dicho concepto. En su reemplazo adviene el "pueblo":

La monarquía absoluta había sido la decisión en la lucha de intereses y coaliciones en conflicto, y con ello había fundado la unidad estatal. La unidad que presenta un pueblo no tiene este carácter decisionista; es una unidad orgánica, y con la conciencia nacional surgen las representaciones del todo orgánico del Estado. Por tal medio tanto el 
concepto teísta como el deísta de Dios se vuelve incomprensible para la metafísica política. (53 / 51)

El resultado final de este proceso queda, pues, a la vista. Schmitt lo resume en "dos momentos característicos: la eliminación de todas las representaciones teístas y trascendentes y la configuración de un nuevo concepto de legitimidad" (54 / 52). Este nuevo concepto, que sustituye al monárquico, es "el pensamiento democrático de la legitimidad" (55 / 52). Es precisamente en este punto que se inscribe para Schmitt la figura y el pensamiento del gran conservador monarquista español Donoso Cortés. ${ }^{15}$

El eclipse del concepto tradicional de legitimidad solo deja como posibilidad, para la preservación del Estado y del orden, la dictadura. Esta era, precisamente, la forma que Schmitt había discutido pacientemente en su gran tratado anterior. En el argumento que ahora presenta, entiende que Donoso, desesperando de toda posibilidad de retorno de la monarquía, habría percibido la situación teorético-política de esa forma en toda su envergadura y urgencia, definida por una disyuntiva sin mediación: entweder-oder, o una democracia plebiscitaria sometida a la inestabilidad propia del populismo, cuyo sujeto fundamental es la burguesía liberal y cuyo mecanismo es la discusión y, si se quiere, en términos de modelo, la acción comunicativa y el ideal

\footnotetext{
${ }^{15}$ Anunciado ya en la conclusión del tercero, Donoso Cortés es el referente principal del cuarto y último capítulo de la Teología política, "Acerca de la filosofía del Estado de la contrarrevolución (De Maistre, Bonald, Donoso Cortés)" (57 ss. / 54 ss.)
} 
de la transparencia-, o la dictadura política, cuyo sujeto es el soberano que decide el estado de excepción a fin de reimplantar el orden, y cuya decisión, enteramente alérgica a toda discusión y todo procedimiento justificatorio, es una "decisión absoluta generada desde la nada" (69/ 62):

\section{Consideraciones finales}

Es casi un lugar común hablar del carácter paradójico del pensamiento de Carl Schmitt. Esta imputación, que sin duda encuentra muchos asideros en su vasta obra, puede ser barajada alegando atenuantes que tampoco la favorecen. Los avatares que ese pensamiento experimenta balanceándose sobre la cresta de la ola histórica que llevó al nazismo al poder y a Schmitt a una eminencia indiscutida en la Alemania de la época, los que tras la derrota parecieran arrollar con él, y hasta aquellos que se han verificado más tarde, también post-mortem, a propósito de su recepción abundante en la discusión política y teórico-política de, digamos, los setenta en adelante, todo ello, en fin, acusa tal vez mucho más un tipo de reflexión cuya consistencia y consecuencia están permanentemente amenazadas por la circunstancia histórica y por la propia posición de su sujeto en ella. Dicho de otro modo, no se puede descontar una fuerte dosis de oportunismo y de acomodo a la circunstancia que, motivando la inhibición o el abandono de tesis y opiniones que antes fueron sustentadas con desplante de convicción, dan la impresión de un pensamiento de vocación 
sistemática que no logra allanarse a las exigencias que esta misma vocación impone, y cae con facilidad en la contradicción.

Pero nuestro asunto no es aquí histórico ni biográfico. Como hemos señalado con insistencia, se ha tratado - en esto que no es sino una primera aproximación a la teoría schmittiana — de discernir la lógica de los conceptos que el autor pone en movimiento para sostener su teoría de la soberanía. En esta fase conclusiva, un juicio provisorio sobre aquel carácter paradójico debe atenerse a los datos que hemos creído poder extraer de nuestra inspección e incidir, precisamente, en dicha lógica.

En este contexto, parece posible apuntar a dos componentes teóricos, que ciertamente han sido señalados por algunos comentaristas de Schmitt, y que atañen a las nociones solidarias de excepción y decisión, en la medida en que estas aparecen acotadas por el prurito esencial del orden.

Por una parte, está, pues, la cuestión de la excepción y la extra-legalidad. Comentando la forma "comisarial" de dictadura que favorece Schmitt ${ }^{16}$ y la condición constitucional que de ella se sigue para el dictador, Paul Hirst anota una paradoja central en su pensamiento:

A despecho de todo su énfasis en las relaciones amigo-enemigo, en la acción política decisoria, su núcleo, su finalidad, es el mantenimiento de

${ }^{16}$ Cf. Schmitt 1928. 
la estabilidad y el orden. Está fundada [su doctrina] en una no-ley política, mas no en el interés de la falta de ley. (Hirst 1999, 14)

Esta exigencia originaria del pensamiento de Schmitt, que está en el fundamento de su concepción jurídica y de su idea del Estado implica una determinación de la excepción que trae consigo una limitación del poder que es propio al estado de excepción, y que bien se acusa en la invocación de que este "siempre es algo distinto a la anarquía y al caos" y en la estipulación de que el soberano, en el ejercicio mismo de la decisión excepcional, pertenece al orden jurídico. Desde el punto de vista lógico, ocurre, pues, como si el principio del orden operase como un correctivo de las consecuencias radicales que trae consigo el pensamiento límite de la excepción y del caso extremo, en el instante mismo en que extrae su posibilidad de él.

Por otra parte, la exigencia del orden conlleva otro aspecto paradójico, en la medida en que es la exigencia de un principio originariamente desprovisto de todo contenido concreto, de toda normatividad sustantiva que - como en el caso de los conservadurismos pre-modernos - pudiera derivarse de la tradición. Un modo particularmente inteligente de apuntar a ese carácter paradójico es el que ensaya Slavoj Žižek:

La paradoja básica del decisionismo político de Carl Schmitt —el régimen de la ley depende en última instancia de un acto abisal de violencia (imposición violenta) que está fundado en sí mismo; esto es, todo orden positivo al que refiera este acto para legitimarse a sí mismo 
es puesto de manera auto-referente por este acto mismo- es que su propia polémica contra el formalismo liberal-democrático cae inexorablemente en la trampa formalista.

[...] La paradoja reside, así, en el hecho de que el único modo de oponerse al formalismo normativo legal es revertir hacia el formalismo decisionista - no hay modo de escapar al formalismo dentro del horizonte de la modernidad. (Žižek 1999, 18 s.)

Pero la textura paradójica del pensamiento de Schmitt es también su fuerza, la capacidad que este tiene para desatar preguntas fundamentales sobre el devenir de lo político y del Estado en la modernidad. Quizá no sea tanto el hecho de que este pensamiento pueda verse invalidado en su consistencia teórica por las paradojas que alberga y que, de hecho, lo movilizan, sino el modo en que él mismo se relaciona con el dinamismo latente en ellas y, si se quiere decirlo así, el no llevar hasta las últimas consecuencias el pensamiento de la excepción y del resto. Una inspección del notable ensayo de Walter Benjamín "Para una crítica de la violencia”, que en tantos aspectos está en debate con la teología política de Schmitt podría arrojar una luz sobre el estilo y las consecuencias de una distinta relación. Pero esto ya es tema de otro desarrollo.

\section{Referencias bibliográficas}

Berndt, Ludwig (2003). "Die Unterscheidung von Freund und Feind als Kriterium des Politischen”. En: Reinhard Mehring (Hg.). Carl Schmitt. 
Der Begriff des Politischen. Ein kooperativer Kommentar. Berlin: Akademie Verlag.

Hirst, Paul. "Carl Schmitt's Decisionism” (1999). En: Chantal Mouffe (ed.), The Challenge of Carl Schmitt. London/New York: Verso.

Müller, Jan-Werner (2003). A Dangerous Mind. Carl Schmitt in PostWar European Thought. New Haven \& London: Yale University Press.

Negretto, Gabriel L. "El concepto de decisionismo en Carl Schmitt. El poder negativo de la excepción”. En: Revista Sociedad, Facultad de Ciencias Sociales, Universidad de Buenos Aires (internet).

Schmitt, Carl (2002). Der Begriff des Politischen. 7. Auflage. 5. Nachdruck der Ausgabe von 1963. Berlin: Duncker \& Humblot.

(1984). El concepto de lo político. Edición preparada por José Aricó. Traducción de Eduardo Molina y Vedia y Raúl Crisafio. Buenos Aires: Folios.

(2004). Politische Theologie. Vier Kapitel zur Lehre von der Souveränität. Achte Auflage. Berlin: Duncker \& Humblot.

$\left(1928^{2}\right)$ Die Diktatur. Von den Anfängen des modernen Souverinitätsgedankens bis zum proletarischen Klassenkampf. München und Leipzig : Duncker \& Humblot. 
[Schmitt, Carl] (2004). Carl Schmitt, Teólogo de la Política. Prólogo y selección de textos de Héctor Orestes Aguilar. México: Fondo de Cultura Económica.

Žižek, Slavoj (1999). "Carl Schmitt in the Age of Post-Politics". En: Chantal Mouffe (ed.), The Challenge of Carl Schmitt. London/New York: Verso, 